\title{
Detection of Delayed Neutrons from Fissionable Samples: Monte Carlo Modelling and Physical Assumptions for a Design of the DET-12 Device
}

\author{
Arkadiusz Kurowski ${ }^{1}$ Barbara Bieńkowska ${ }^{2} \cdot \mathrm{Krzysztof} \mathrm{Drozdowicz}^{1} \cdot$ Barbara Gabańska $^{1} \cdot$ Andrzej Igielski $^{1} \cdot$ \\ Rafał Prokopowicz ${ }^{3} \cdot$ Grzegorz Tracz $^{1} \cdot$ Urszula Wiącek $^{1} \cdot$ Urszula Woźnicka $^{1}$
}

Published online: 29 March 2018

(C) The Author(s) 2018

\begin{abstract}
An activation of fissionable materials with neutrons has been considered as a possible neutron diagnostic of D-D and D-T fusion plasma. Fission reaction caused by fusion neutrons leads up to emission of secondary neutrons: prompt and delayed. Physical assumptions have been outlined to design a new device (DET-12) for measurements of delayed neutrons emitted from samples of fissionable materials activated with neutrons at big fusion-plasma devices. The aim is to support a classic neutron activation method used as one of plasma diagnostics at tokamaks or stellarators. An interpretation of the time decay of delayed neutrons enables an assessment of the primary neutron flux which induced fission reaction. Monte Carlo calculations have been carried out in order to elaborate the method considered. Nuclides like: pure ${ }^{235} \mathrm{U},{ }^{238} \mathrm{U}$ and ${ }^{232} \mathrm{Th}$, have been selected as possible materials to be irradiated. Physical fundamentals of generation of the delayed neutrons are mentioned and a resulting concept of the DET-12 device, built in the Institute of Nuclear Physics, Poland, is presented. A general size and dimensions of particular constituent material layers, and a number and placement of neutron detectors are optimized by means of Monte Carlo modelling. Recommendations for a technical design of the measuring chamber were formulated. Detection efficiency of DET-12 has been also estimated.
\end{abstract}

Keywords Nuclear fusion diagnostics · Neutron diagnostics · Neutron activation method · Fission reaction · Delayed neutrons

\section{Introduction}

The neutron activation method is one of the standard methods for the plasma diagnostics at tokamaks. The activation detectors (targets of chosen isotopes) are placed in exposition locations, where the neutron yield from fusion plasma (D-D or D-T) is measured. After the activation the samples are delivered with a pneumatic transport system to a gamma-ray spectrometer. A deconvolution of

Arkadiusz Kurowski

Arkadiusz.Kurowski@ifj.edu.pl

1 Institute of Nuclear Physics PAN, ul. Radzikowskiego 152, 31-342 Kraków, Poland

2 Institute of Plasma Physics and Laser Microfusion, ul. Hery 23, 01-497 Warsaw, Poland

3 National Centre for Nuclear Research, ul. A. Sołtana 7, 05-400 Otwock-Świerk, Poland the spectra allows calculating the primary neutron yield in given points.

A valuable supplement to the conventional neutron activation method is a delayed neutron activation method (e.g. at JET tokamak [1]) which uses fissionable isotopes as activated targets. Fissionable isotopes as ${ }^{235} \mathrm{U},{ }^{238} \mathrm{U}$ or ${ }^{232} \mathrm{Th}$, irradiated with neutrons, emit prompt neutrons $(\sim 99 \%)$ and later the so-called delayed neutrons $(\sim 1 \%)$ from the fission products which decay with the betaemission. The decay chain can be generally written as

${ }_{\mathrm{Z}}^{\mathrm{A}} \mathrm{X}_{\mathrm{N}} \stackrel{\beta}{\longrightarrow}{ }_{\mathrm{Z}+1}^{\mathrm{A}} \mathrm{Y}_{\mathrm{N}-1} \rightarrow{ }_{\mathrm{Z}+1}^{\mathrm{A}} \mathrm{Y}_{\mathrm{N}-2}^{\prime}+\mathrm{n}$

where the $\mathrm{X}$ nucleus is the delayed neutron precursor and $\mathrm{Y}$ is the delayed neutron emitter. The delay is determined by the half-life time of the precursor. Usually, six groups of the delayed neutrons (originated by different fission products) are distinguished [2] and the total decay in time is described by 
$S(t)=r_{\mathrm{f}} \sum_{i=1}^{6} v_{\mathrm{d} i} \lambda_{\mathrm{d} i} \exp \left(-\lambda_{\mathrm{d} i} t\right)$

where $r_{\mathrm{f}}$ is the number of fission reactions in the sample, $v_{\mathrm{d} i}$ is the yield of the $i$ th delayed neutron group, $\lambda_{\mathrm{d} i}$ is the decay constant of this group. The corresponding half-life time can range in some cases up to 1 or $2 \mathrm{~min}$. For example, the ${ }^{235} \mathrm{U}$ fission products being the precursors for the delayed neutrons, have the average half-life times from $\sim 0.2$ to $\sim 56 \mathrm{~s}$. The average energy of the delayed neutrons is in the range of $(300-600 \mathrm{keV})[2,3]$.

\section{Conceptual Design of the Device and Modelling of the Radiation Transport}

A system of the detection of delayed neutrons must have a high sensitivity for delayed neutrons escaping from the fissionable sample and a low sensitivity for accompanied $\gamma$ radiation which can enter the counters. In our design a number of thermal neutron detectors $\left({ }^{3} \mathrm{He}\right.$ type), are placed in a neutron moderator, and surrounded a central cavity for the sample (Fig. 1). The cavity is surrounded by a bismuth layer which prevents counters from $\gamma$ radiation. The next polyethylene layer which contains ${ }^{3} \mathrm{He}$ detectors is used to slow down delayed neutrons to thermal energies. The external layers consisting of polyethylene, cadmium, and a $\mathrm{B}_{4} \mathrm{C}$, prevent outside background neutrons entering the detectors.

Detectors are connected to separate electronic lines (H.V. supply, preamplifier, amplifier, counting electronics) and finally to a control PC unit with the data acquisition and analysing systems.
All elements of the experimental set-up have been optimized by detailed Monte Carlo calculations, i.e. type of the fissionable material, geometry and size of the target sample, number and location of ${ }^{3} \mathrm{He}$ detectors, shape and sizes of all covers. The set-up has been modelled using MCNP numerical code for radiation transport [4] and details of calculations are presented in the reports $[3,5,6]$.

DET-12 device has been optimized for measurement of delayed neutrons produced during fission reactions of ${ }^{235} \mathrm{U}$ induced by thermal and fast neutrons and of ${ }^{238} \mathrm{U}$ and ${ }^{232} \mathrm{Th}$ induced by fast neutrons. These two last nuclides are characterized by energy threshold for neutron-induced fission reaction (about $1 \mathrm{MeV}$ for ${ }^{238} \mathrm{U}$ and $0.5 \mathrm{MeV}$ for ${ }^{232} \mathrm{Th}$ ) which is a valuable feature for the detection of fast neutrons.

Size and shape of the target sample were optimized taking into account the typical shape of the pneumatic system applied at JET [1] and a magnitude of neutron current through the sample surface. The cylindrical sample of height $1.8 \mathrm{~cm}$ and diameter $1.8 \mathrm{~cm}$ has been chosen.

Twelve ${ }^{3} \mathrm{He}$ neutron detectors: $1^{\prime \prime}$ diameter, $30 \mathrm{~cm}$ length ( $25 \mathrm{~cm}$ active), $5 \mathrm{~atm}$. pressure have been chosen as the optimum number. The total efficiency of detection, calculated as a sum from all 12 detectors has been evaluated from the MCNP simulations on $24 \%$. The simulations were performed either for all 16 detectors or for 12 detectors, i.e. when the detectors in corners are removed (Fig. 1b). The contribution of these detectors is smaller than of the others. The efficiency decreases less than it results only from the ratio of $12 / 16$. As expected MCNP calculations confirmed, the contribution of the corner detectors is smaller than of the others. A decrease of the efficiency, ratio $\varepsilon(\mathrm{N} 12) / \varepsilon(\mathrm{N} 16) \approx 0.87$, is smaller than (a)

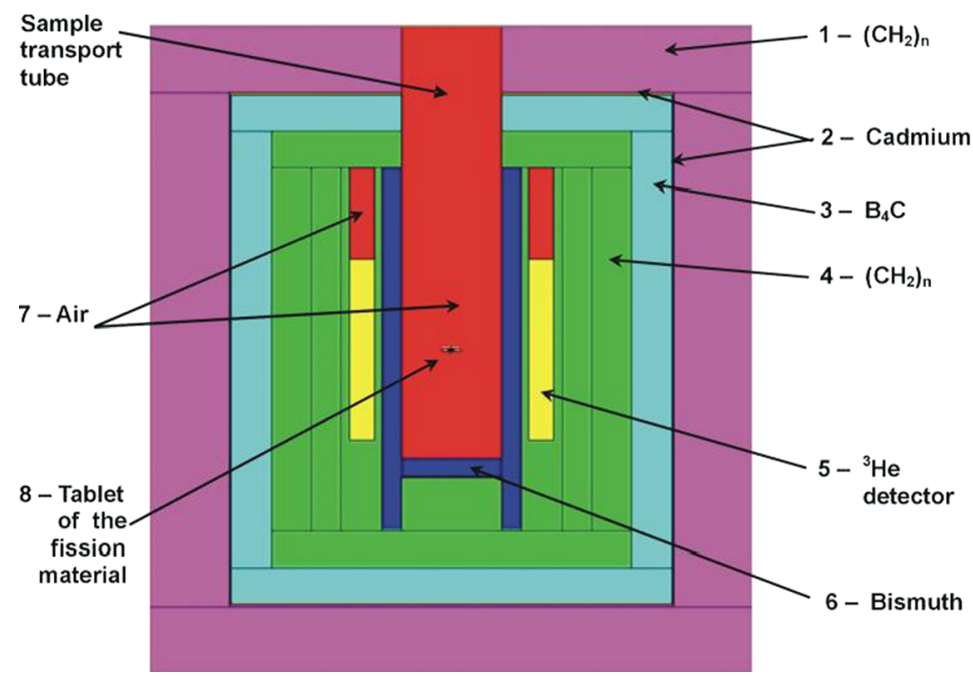

(b)

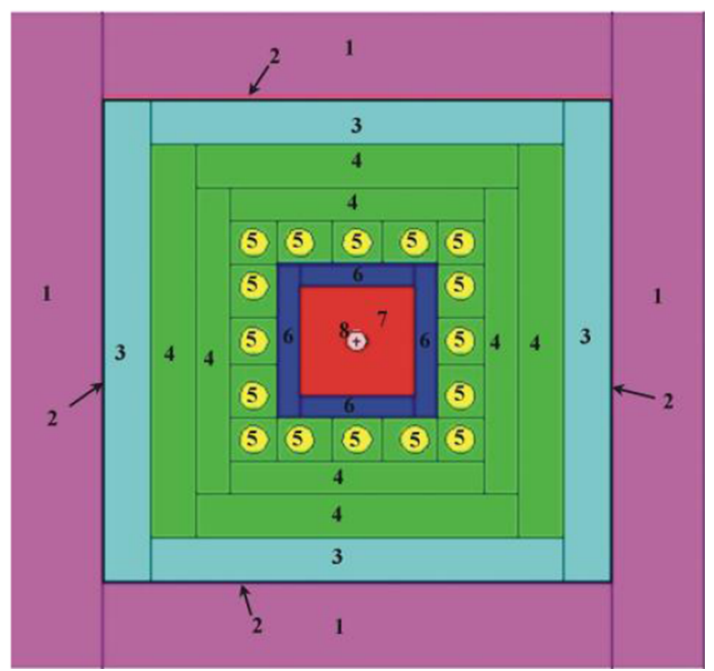

Fig. 1 MCNP model of the DET-12 device (vertical and horizontal cross sections) [5]. a Vertical section. b Horizontal section 
that which would result from the ratio of numbers of the detectors, $12 / 16=0.75$.

Final dimensions for the optimized DET-12 device are:

- Square horizontal size: $58 \mathrm{~cm} \times 58 \mathrm{~cm}$, height $74 \mathrm{~cm}$

- Central hole for the pneumatic transport: $6 \mathrm{~cm} \times 6 \mathrm{~cm}$

- Consecutive layers from the hole towards outside (see Fig. 1): (6) bismuth: $2 \mathrm{~cm}$, (4) polyethylene (moderator of delayed neutrons): $12 \mathrm{~cm}$, (3) $\mathrm{B}_{4} \mathrm{C}$ (absorber): $3.8 \mathrm{~cm}$, (2) Cadmium (absorber): $0.2 \mathrm{~cm}$, (1) Polyethylene (external protection): $8 \mathrm{~cm}$.

\section{Electronic System for Neutron Detection and Data Acquisition}

The expected intensity of counts in the ${ }^{3} \mathrm{He}$ detectors will be rather low. Therefore, it has been decided to use a system in which three ${ }^{3} \mathrm{He}$ detectors are coupled to one preamplifier. Then the preamplifiers in a usual way are connected to spectroscopy amplifiers. Four lines are connected with a digitizer card in a PC. A complete scheme of the system is shown in Fig. 2 [5]. All the electronic blocks were connected (including a preparation of dedicated cables for the groups of detectors) after preparatory operation tests and H.V. and amplification adjustments were made.

\section{Detection Efficiency of DET-12 Device [5]}

Detection efficiency of the DET-12 device was tested using the ${ }^{252} \mathrm{Cf}$ source of activity $=8.777 \mathrm{kBq}$ placed in the middle of the measuring channel of DET-12, i.e. in the position presumed for the activated sample to be measured. Spontaneous fission of ${ }^{252} \mathrm{Cf}$ brings the neutron emission with the coefficient of $11.627 \%$ [7]. This results in the neutron emission rate of $1020 \mathrm{cps}$. The obtained average count rate of the DET-12 device is $191.4 \pm 0.2 \mathrm{cps}$. Thus, the neutron detection efficiency can be assessed at $18.8 \%$. Comparing the experimental results with those obtained from the Monte Carlo simulations the difference of the estimations is $20 \%$. Probable reason may be inaccurate knowledge of the active volume of the detector and its gas composition, which were used for numerical calculations. The Monte Carlo simulations do not include neither dead time nor efficiency of the detectors (problem of the detector active volume). Real composition of the gas in the detectors may be different from the one assumed in the MC simulations-manufacturer does not release even principal information and additionally some admixtures, like $\mathrm{CO}_{2}$ or $\mathrm{Ar}$ as a quench gas, (below 1\%) are generally known to be present.

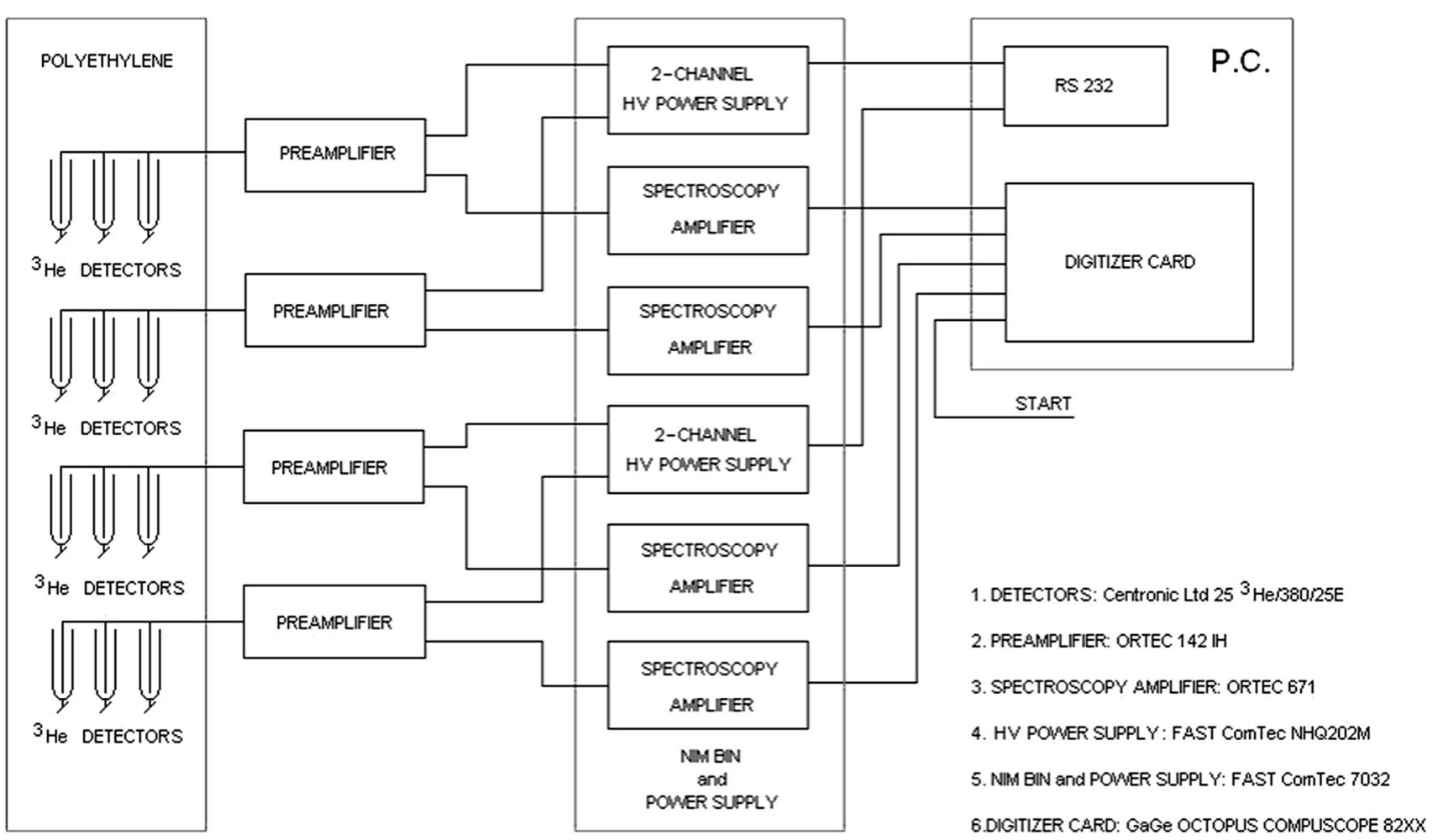

Fig. 2 Neutron detection and data acquisition system [5] 


\section{Summary}

Measurement of delayed neutrons which are emitted from fissionable samples irradiated in a neutron field originated from thermonuclear plasma is one of types of the neutron activation method applicable for a neutron diagnostic of fusion plasma.

The experimental device (DET-12) has been designed, optimized and constructed for the measurements of delayed neutrons from the neutron induced fission reactions. A small amount of delayed neutrons generated during the fission reactions dedicates this method to the measurement of very high neutron yields generated in large fusion devices. Analytical methods, MCNP calculations and experiments indicate that DET-12 device can works with good statistics with minimal neutron fluxes about $10^{8}-10^{9}$ $\mathrm{n} / \mathrm{cm}^{2} \mathrm{~s}$.

Open Access This article is distributed under the terms of the Creative Commons Attribution 4.0 International License (http://creative commons.org/licenses/by/4.0/), which permits unrestricted use, distribution, and reproduction in any medium, provided you give appropriate credit to the original author(s) and the source, provide a link to the Creative Commons license, and indicate if changes were made.

\section{References}

1. O.N. Jarvis, E.W. Clipsham, M.A. Hone, B.J. Laundy, M. Piullon, M. Rapisarda, G. Sadler, P. van Belle, K.A. Verschuur, Fusion Technol. 20, 265-284 (1991)

2. G.R. Keepin, T.F. Wiemett, R.K. Zeigler, Phys. Rev. 107, 1044-1049 (1957)

3. B. Bieńkowska, K. Drozdowicz, B. Gabańska, A. Igielski, R. Prokopowicz, U. Wiącek, U. Woźnicka, Rept. IFJ No. 2074/AP, 2014, Kraków

4. X-5 Monte Carlo Team, MCNP, Los Alamos National Laboratory, LA-UR-03-1987, (2003)

5. B. Bieńkowska, K. Drozdowicz, B.Gabańska, A. Igielski, W. Janik, A. Kurowski, G. Tracz, U. Wiącek, J. Dankowski, Rept. IFJ No. 2075/AP, 2014, Kraków

6. G. Tracz, B. Bieńkowska, K. Drozdowicz, Rept. IFJ No. 2061/PN, 2013, Kraków

7. M.-M. Be, V. Chiste, in Table of Radionuclides, vol. 4, (2008), p. 277. http://www.bipm.org/utils/common/pdf/monographieRI/ Monographie_BIPM-5_Tables_Vol4.pdf 